\title{
Completely integrable sector in 5D Einstein-Maxwell gravity and derivation of the dipole black ring solutions
}

\author{
Stoytcho S. Yazadjiev * \\ Department of Theoretical Physics, Faculty of Physics, Sofia University, \\ 5 James Bourchier Boulevard, Sofia 1164, Bulgaria
}

\begin{abstract}
We consider 5D Einstein-Maxwell (EM) gravity in spacetimes with three commuting Killing vectors: one timelike and two spacelike Killing vectors one of them being hypersurface-orthogonal. Assuming a special ansatz for the Maxwell field we show that the 2-dimensional reduced EM equations are completely integrable by deriving a Lax-pair presentation. We also develop a solution generating method for explicit construction of exact EM solutions with considered symmetries. We also derive explicitly a new rotating six parametric 5D EM solution which includes the dipole black ring solution as a particular case.
\end{abstract}

\section{Introduction}

In recent years the higher dimensional gravity is attracting much interest. Apart from the fact that the higher dimensional gravity is interesting in its own right, the increasing amount of works devoted to the study of the higher dimensional spacetimes is inspired from the string theory and the brane-world scenario with large extra dimensions. The gravity in higher dimensions exhibits much richer dynamics and spectrum of solutions than in four dimensions. One of the most reliable routes for better understanding of higher dimensional gravity and the related topics are the exact solutions. For example, recently discovered exact black rings solutions with unusual horizon topology 1, 2], demonstrated explicitly that the 5D Einstein gravity exhibits unexpected features completely absent in four dimensions. It was shown in [2] that both the black hole and the the black ring can carry the same conserved charges, the mass and a single angular momentum, and therefore there is no uniqueness theorem in five dimensions. Moreover, the black rings can also carry nonconserved charges which can be varied continuously without altering the conserved charges. This fact leads to continuous (classical) non-uniqness [3].

*E-mail: yazad@phys.uni-sofia.bg 
The higher dimensional solutions found so far are not so many. As yet to the best of our knowledge there are no EM solutions found in the literature that describe rotating charged black holes in higher dimensions. However, some numerical solutions were recently constructed in [4 (see also [5]). Moreover the systematic construction of new solutions in higher dimensions has not been accomplished in comparison with $4 \mathrm{D}$ case. It is well known that both vacuum and electrovacuum 4D Einstein equations are completely integrable being restricted to spacetimes with two-dimensional Abelian group of isometries [6]- 20]. This nice property is also shared by some effective string gravity models (or certain sectors of them) which allows us to find many families of physically interesting exact solutions [21]-26]. The $D$-dimensional vacuum Einstein equations with $(D-2)$-dimensional Abelian group of isometries are completely integrable, too 27, 28.

The aim of this work is to make a step in systematic construction of exact solutions in 5D EM gravity. We show here that a certain sector of EM gravity is completely integrable. We also present an explicit method for generating exact 5D EM solutions from known solutions of the 5D vacuum Einstein equations. As an illustration of the method we derive explicitly a new rotating six parametric 5D EM solution which includes the dipole black ring solution as a particular case.

\section{Dimensional reduction, coset presentation and complete integrability}

The 5D EM gravity is described by the field equations

$$
\begin{aligned}
& R_{\mu \nu}=\frac{1}{2}\left(F_{\mu \lambda} F_{\nu}^{\lambda}-\frac{1}{6} F_{\sigma \lambda} F^{\sigma \lambda} g_{\mu \nu}\right), \\
& \nabla_{\mu} F^{\mu \nu}=0 .
\end{aligned}
$$

In this paper we consider 5D EM gravity in spacetimes with three commuting Killing vectors: one timelike Killing vector $T$ and two spacelike Killing vectors $K_{1}$ and $K_{2}$. We also assume that the Killing vector $K_{2}$ is hypersurface orthogonal.

In adapted coordinates in which $K_{2}=\partial / \partial Y$, the spacetime metric can be written into the form

$$
d s^{2}=e^{2 u} d Y^{2}+e^{-u} h_{i j} d x^{i} d x^{j}
$$

where $h_{i j}$ is a 4 -dimensional metric with Lorentz signature. Both $u$ and $h_{i j}$ depend on the coordinates $x^{i}$ only. The electromagnetic field is taken in the form ${ }^{1}$

$$
F=d A_{Y} \wedge d Y .
$$

After a dimensional reduction along the Killing vector $K_{2}$, the field equations (11) are reduced to the following effective $4 \mathrm{D}$ theory:

\footnotetext{
${ }^{1}$ Throughout this paper we denote the Killing vectors and their naturally corresponding 1-forms by the same letter.
} 


$$
\begin{aligned}
& \mathcal{D}_{i} \mathcal{D}^{i} u=-\frac{1}{3} e^{-2 u} h^{i j} \mathcal{D}_{i} A_{Y} \mathcal{D}_{j} A_{Y}, \\
& \mathcal{D}_{i}\left(e^{-2 u} \mathcal{D}^{i} A_{Y}\right)=0, \\
& R(h)_{i j}=\frac{3}{2} \partial_{i} u \partial_{j} u+\frac{1}{2} e^{-2 u} \partial_{i} A_{Y} \partial_{j} A_{Y} .
\end{aligned}
$$

Here $\mathcal{D}_{i}$ and $R(h)_{i j}$ are the covariant derivative and Ricci tensor with respect to the Lorentz metric $h_{i j}$. Let us introduce the symmetric matrix $M_{1}$ given by

$$
M_{1}=\left(\begin{array}{cc}
e^{u}+\frac{1}{3} e^{-u} A_{Y}^{2} & \frac{1}{\sqrt{3}} e^{-u} A_{Y} \\
\frac{1}{\sqrt{3}} e^{-u} A_{Y} & e^{-u}
\end{array}\right)
$$

with $\operatorname{det} M_{1}=1$. Then the dimensionally reduced EM equations become

$$
\begin{aligned}
& \mathcal{D}_{i}\left[\mathcal{D}^{i} M_{1} M_{1}^{-1}\right]=0, \\
& R_{i j}(h)=-\frac{3}{4} \operatorname{Tr}\left[\partial_{i} M_{1} \partial_{j} M_{1}^{-1}\right] .
\end{aligned}
$$

These equations are yielded by the action

$$
S=\frac{1}{16 \pi} \int d^{4} x \sqrt{h}\left[R(h)+\frac{3}{4} h^{i j} \operatorname{Tr}\left(\partial_{i} M_{1} \partial_{j} M_{1}^{-1}\right)\right] .
$$

Clearly the action is invariant under the $S L(2, R)$ group where the group action is given by

$$
M_{1} \rightarrow G M_{1} G^{T}
$$

$G \in S L(2, R)$. In fact the matrices $M_{1}$ parameterize a $S L(2, R) / S O(2)$ coset. So we obtain non-linear $\sigma$-model coupled to $4 \mathrm{D}$ Einstein gravity.

Next step is to further reduce the effective $4 D$ theory along the Killing vectors $T$ and $K_{1}$. In this connection it is useful to introduce the twist $\omega$ of the Killing vector $T$ defined by

$$
\omega=\frac{1}{2} \star(h)(T \wedge d T)
$$

were $\star(h)$ is the Hodge dual with respect to the metric $h_{i j}$.

One can show that the Ricci 1-form $\Re_{h}[T]$ defined by

$$
\Re_{h}[T]=R_{i j}(h) T^{j} d x^{i},
$$

satisfies 


$$
\star(h)\left(T \wedge \Re_{h}[T]\right)=d \omega .
$$

Obviously, in our case we have $\Re_{h}[T]=0$, i.e. $d \omega=0$. Therefore there exists (locally) a potential $f$ such that

$$
\omega=d f
$$

In adapted coordinates for the Killing vectors $T=\partial / \partial t$ and $K_{1}=\partial / \partial X$, and in the canonical coordinates $\rho$ and $z$ for the transverse space, the $4 \mathrm{D}$ metric $h_{i j}$ can be written into the form

$$
h_{i j} d x^{i} d x^{j}=-e^{2 U}(d t+\mathcal{A} d X)^{2}+e^{-2 U} \rho^{2} d X^{2}+e^{-2 U} e^{2 \Gamma}\left(d \rho^{2}+d z^{2}\right) .
$$

For this form of the metric $h_{i j}$, combining (12) and (15), and after some algebra we find that the twist potential $f$ satisfies

$$
\begin{aligned}
\partial_{\rho} f & =-\frac{1}{2} \frac{e^{4 U}}{\rho} \partial_{z} \mathcal{A}, \\
\partial_{z} f & =\frac{1}{2} \frac{e^{4 U}}{\rho} \partial_{\rho} \mathcal{A} .
\end{aligned}
$$

Before writing the $2 \mathrm{D}$ reduced equations we shall introduce the symmetric matrix

$$
M_{2}=\left(\begin{array}{cc}
e^{2 U}+4 f^{2} e^{-2 U} & 2 f e^{-2 U} \\
2 f e^{-2 U} & e^{-2 U}
\end{array}\right)
$$

with $\operatorname{det} M_{2}=1$. Then the $2 \mathrm{D}$ reduced EM equations read

$$
\begin{aligned}
& \partial_{\rho}\left(\rho \partial_{\rho} M_{1} M_{1}^{-1}\right)+\partial_{z}\left(\rho \partial_{z} M_{1} M_{1}^{-1}\right)=0, \\
& \partial_{\rho}\left(\rho \partial_{\rho} M_{2} M_{2}^{-1}\right)+\partial_{z}\left(\rho \partial_{z} M_{2} M_{2}^{-1}\right)=0, \\
\rho^{-1} \partial_{\rho} \Gamma=- & -\frac{1}{8}\left[\operatorname{Tr}\left(\partial_{\rho} M_{2} \partial_{\rho} M_{2}^{-1}\right)-\operatorname{Tr}\left(\partial_{z} M_{2} \partial_{z} M_{2}^{-1}\right)\right] \\
& -\frac{3}{8}\left[\operatorname{Tr}\left(\partial_{\rho} M_{1} \partial_{\rho} M_{1}^{-1}\right)-\operatorname{Tr}\left(\partial_{z} M_{1} \partial_{z} M_{1}^{-1}\right)\right], \\
\rho^{-1} \partial_{z} \Gamma= & -\frac{1}{4} \operatorname{Tr}\left(\partial_{\rho} M_{2} \partial_{z} M_{2}^{-1}\right) \\
& -\frac{3}{4} \operatorname{Tr}\left(\partial_{\rho} M_{1} \partial_{z} M_{1}^{-1}\right) .
\end{aligned}
$$

As a result we find that that the "field variables" $M_{1}$ and $M_{2}$ satisfy the equations of two $S L(2, R) / S O(2) \sigma$-models in two dimensions, modified by the presence of the factor $\rho$. The system equations for $\Gamma$ can be integrated, once a pair of solutions for the 
two $\sigma$-models is known. Therefore, the problem of generating solutions to the 5D EM equations with the described symmetries reduces to the solutions of the two $\sigma$-models.

It is well-known that the $\sigma$-model equations are completely integrable [29, 30]. This is a consequence of the fact that the $\sigma$-model equations can be considered as the compatibility condition of the linear differential equations (Lax-pair presentation) 29. 30

$$
\begin{aligned}
D_{\rho} \Psi & =\frac{\rho \mathcal{U}+\lambda V}{\lambda^{2}+\rho^{2}} \Psi \\
D_{z} \Psi & =\frac{\rho V-\lambda \mathcal{U}}{\lambda^{2}+\rho^{2}} \Psi
\end{aligned}
$$

where

$$
D_{\rho}=\partial_{\rho}+\frac{2 \lambda \rho}{\lambda^{2}+\rho^{2}} \partial_{\lambda}, \quad D_{z}=\partial_{z}-\frac{2 \lambda^{2}}{\lambda^{2}+\rho^{2}} \partial_{\lambda}
$$

Here $V=\rho \partial_{z} M M^{-1}, \mathcal{U}=\rho \partial_{\rho} M M^{-1}$ and $\lambda$ is the complex spectral parameter. The "wave function" $\Psi(\rho, z, \lambda)$ is a complex matrix. The $\sigma$-model equations then follows from the compatibility condition

$$
\left[D_{\rho}, D_{z}\right] \Psi=0
$$

The matrix $M$ can be found from the "wave function" $\Psi$ as $M(\rho, z)=\Psi(\rho, z, \lambda=0)$.

The inverse scattering transform (IST) method can be directly applied to (24) to generate multisoliton solutions. The dressing procedure allows us to generate new solutions from known ones. Since this dressing technique is well known we will not discuss it here and refer the reader to [29, 30].

In this paper we will not apply the IST method. In the next section we present new and simple enough solution generating method which allows us to generate new 5D EM solutions from known solutions of the 5D vacuum Einstein equations.

\section{Solution construction}

Let us consider two solutions $M_{1}=M^{(1)}$ and $M_{2}=M^{(2)}$ of the $\sigma$-model equations

$$
\partial_{\rho}\left(\rho \partial_{\rho} M M^{-1}\right)+\partial_{z}\left(\rho \partial_{z} M M^{-1}\right)=0
$$

In addition let us denote by $\gamma^{(i)}$ the solution of the system

$$
\begin{aligned}
\rho^{-1} \partial_{z} \gamma^{(i)} & =-\frac{1}{4} \operatorname{Tr}\left(\partial_{\rho} M^{(i)} \partial_{z} M^{(i)}-1\right. \\
\rho^{-1} \partial_{\rho} \gamma^{(i)} & =-\frac{1}{8}\left[\operatorname{Tr}\left(\partial_{\rho} M^{(i)} \partial_{\rho} M^{(i)^{-1}}\right)-\operatorname{Tr}\left(\partial_{z} M^{(i)} \partial_{z} M^{(i)}-1\right)\right] .
\end{aligned}
$$


Then we find for the metric function $\Gamma$

$$
\Gamma=\gamma^{(2)}+3 \gamma^{(1)}
$$

From a practical point of view it is more convenient to associate the $\sigma$-model solutions $M^{(i)}$ with solutions of the vacuum Einstein equations ${ }^{2}$

$$
\begin{aligned}
d s_{E(i)}^{2}= & e^{2 u_{E}^{(i)}} d Y^{2}+e^{-u_{E}^{(i)}}\left[-e^{2 U_{E}^{(i)}}\left(d t+\mathcal{A}_{E}^{(i)} d X\right)^{2}\right. \\
& \left.+e^{-2 U_{E}^{(i)}} \rho^{2} d X^{2}+e^{-2 U_{E}^{(i)}} e^{2 \Gamma_{E}^{(i)}}\left(d \rho^{2}+d z^{2}\right)\right]
\end{aligned}
$$

which correspond to the matrixes

$$
M^{(i)}=\left(\begin{array}{cc}
e^{2 U_{E}^{(i)}}+4\left(f_{E}^{(i)}\right)^{2} e^{-2 U_{E}^{(i)}} & 2 f_{E}^{(i)} e^{-2 U_{E}^{(i)}} \\
2 f_{E}^{(i)} e^{-2 U_{E}^{(i)}} & e^{-2 U_{E}^{(i)}}
\end{array}\right) .
$$

The metric function $\Gamma_{E}^{(i)}$ for the vacuum Einstein equations can be found from the equations of $\Gamma$ by setting $A_{Y}=0$ in the matrix $M_{1}$. So we obtain

$$
\Gamma_{E}^{(i)}=\gamma^{(i)}+\Omega_{E}^{(i)}
$$

where $\Omega_{E}^{(i)}$ is a solution to the system

$$
\begin{aligned}
\rho^{-1} \partial_{\rho} \Omega_{E}^{(i)} & =\frac{3}{4}\left[\left(\partial_{\rho} u_{E}^{(i)}\right)^{2}-\left(\partial_{z} u_{E}^{(i)}\right)^{2}\right], \\
\rho^{-1} \partial_{z} \Omega_{E}^{(i)} & =\frac{3}{2} \partial_{\rho} u_{E}^{(i)} \partial_{z} u_{E}^{(i)} .
\end{aligned}
$$

We then find from (30) and (33) that

$$
\Gamma=\Gamma_{E}^{(2)}-\Omega_{E}^{(2)}+3\left[\Gamma_{E}^{(1)}-\Omega_{E}^{(1)}\right] .
$$

Comparing the matrixes $M_{1}$ and $M^{(1)}$ we obtain

$$
\begin{gathered}
e^{2 u}=e^{4 U_{E}^{(1)}}, \\
A_{Y}=2 \sqrt{3} f_{E}^{(1)},
\end{gathered}
$$

where $f_{E}^{(i)}$ satisfies $^{3}$

$$
\begin{aligned}
\partial_{\rho} f_{E}^{(i)} & =-\frac{1}{2} \frac{e^{4 U_{E}^{(i)}}}{\rho} \partial_{z} \mathcal{A}_{E}^{(i)} \\
\partial_{z} f_{E}^{(i)} & =\frac{1}{2} \frac{e^{4 U_{E}^{(i)}}}{\rho} \partial_{\rho} \mathcal{A}_{E}^{(i)}
\end{aligned}
$$

\footnotetext{
${ }^{2}$ From now on all quantities with subscript or superscript "E" correspond to the vacuum case.

${ }^{3}$ Clearly, these equations are restriction of (39) to the vacuum case.
} 
Once having the metric function $e^{2 u}=g_{Y Y}$ we can write the EM metric

$$
\begin{array}{r}
d s^{2}=e^{4 U_{E}^{(1)}} d Y^{2}+e^{-2 U_{E}^{(1)}} \\
{\left[-e^{2 U_{E}^{(2)}}\left(d t+\mathcal{A}_{E}^{(2)} d X\right)^{2}+e^{-2 U_{E}^{(2)}} \rho^{2} d X^{2}\right.} \\
\left.+\left(\frac{e^{2 \Gamma_{E}^{(1)}}}{e^{2 \Omega_{E}^{(1)}+\frac{2}{3} \Omega_{E}^{(2)}}}\right)^{3} e^{-2 U_{E}^{(2)}} e^{2 \Gamma_{E}^{(2)}}\left(d \rho^{2}+d z^{2}\right)\right]
\end{array}
$$

Taking into account that

$$
\begin{aligned}
& g_{00}^{E(i)}=-e^{-u_{E}^{(i)}} e^{2 U_{E}^{(i)}} \\
& \tilde{g}_{X X}^{E(i)}=g_{X X}^{E(i)}-g_{00}^{E(i)}\left(\mathcal{A}_{E}^{(i)}\right)^{2}=e^{-u_{E}^{(i)}} e^{-2 U_{E}^{(i)}} \rho^{2}, \\
& g_{\rho \rho}^{E(i)}=e^{-u_{E}^{(i)}} e^{-2 U_{E}^{(i)}} e^{2 \Gamma_{E}^{(i)}}
\end{aligned}
$$

and

$$
\begin{aligned}
e^{4 U_{E}^{(i)}} & =\left(g_{00}^{E(i)}\right)^{2} g_{Y Y}^{E(i)}, \\
e^{2 \Gamma_{E}^{(i)}} & =\left|g_{00}^{E(i)}\right| g_{Y Y}^{E(i)} g_{\rho \rho}^{E(i)},
\end{aligned}
$$

the metric can be presented in more elegant form

$$
\begin{aligned}
d s^{2}= & {\left[\left|g_{00}^{E(1)}\right|{\sqrt{g_{Y Y}^{E(1)}}}^{2} d Y^{2}+\left[\frac{\sqrt{g_{Y Y}^{E(2)}}}{\left|g_{00}^{E(1)}\right| \sqrt{g_{Y Y}^{E(1)}}}\right]\left[g_{00}^{E(2)}\left(d t+\mathcal{A}_{E}^{(2)} d X\right)^{2}+\tilde{g}_{X X}^{E(2)} d X^{2}\right.\right.} \\
& \left.+\left(\frac{\left|g_{00}^{E(1)}\right| g_{Y Y}^{E(1)} g_{\rho \rho}^{E(1)}}{e^{2 \Omega_{E}^{(1)}+\frac{2}{3} \Omega_{E}^{(2)}}}\right)^{3} g_{\rho \rho}^{E(2)}\left(d \rho^{2}+d z^{2}\right)\right]
\end{aligned}
$$

Summarizing, we obtain the following important result formulated as a proposition.

Proposition. Let us consider two solutions of the vacuum 5D Einstein equations

$$
d s_{E(i)}^{2}=g_{Y Y}^{E(i)} d Y^{2}+g_{00}^{E(i)}\left(d t+\mathcal{A}_{E}^{(i)} d X\right)^{2}+\tilde{g}_{X X}^{E(i)} d X^{2}+g_{\rho \rho}^{E(i)}\left(d \rho^{2}+d z^{2}\right)
$$

Then the following give a solution to the 5D EM equations ${ }^{4}$

$$
\begin{aligned}
d s^{2}= & {\left[\left|g_{00}^{E(1)}\right|{\sqrt{g_{Y Y}^{E(1)}}}^{2} d Y^{2}+\left[\frac{\sqrt{g_{Y Y}^{E(2)}}}{\left|g_{00}^{E(1)}\right| \sqrt{g_{Y Y}^{E(1)}}}\right]\left[g_{00}^{E(2)}\left(d t+\mathcal{A}_{E}^{(2)} d X\right)^{2}+\tilde{g}_{X X}^{E(2)} d X^{2}\right.\right.} \\
& \left.+\left(\frac{\left|g_{00}^{E(1)}\right| g_{Y Y}^{E(1)} g_{\rho \rho}^{E(1)}}{e^{2 \Omega_{E}^{(1)}+\frac{2}{3} \Omega_{E}^{(2)}}}\right)^{3} g_{\rho \rho}^{E(2)}\left(d \rho^{2}+d z^{2}\right)\right] \\
A_{Y}= & 2 \sqrt{3} f_{E}^{(1)}
\end{aligned}
$$

${ }^{4}$ More generally we can take $A_{Y}= \pm 2 \sqrt{3} f_{E}^{(1)}+$ const which is obvious. 
where $f_{E}^{(1)}$ is a solution to the system

$$
\begin{aligned}
\partial_{\rho} f_{E}^{(1)} & =-\frac{1}{2} \frac{\left(g_{00}^{E(1)}\right)^{2} g_{Y Y}^{E(1)}}{\rho} \partial_{z} \mathcal{A}_{E}^{(1)}, \\
\partial_{z} f_{E}^{(1)} & =\frac{1}{2} \frac{\left(g_{00}^{E(1)}\right)^{2} g_{Y Y}^{E(1)}}{\rho} \partial_{\rho} \mathcal{A}_{E}^{(1)},
\end{aligned}
$$

and $\Omega_{E}^{(i)}$ satisfy

$$
\begin{aligned}
\rho^{-1} \partial_{\rho} \Omega_{E}^{(i)} & =\frac{3}{16}\left[\left(\partial_{\rho} \ln \left(g_{Y Y}^{E(i)}\right)\right)^{2}-\left(\partial_{z} \ln \left(g_{Y Y}^{E(i)}\right)\right)^{2}\right] \\
\rho^{-1} \partial_{z} \Omega_{E}^{(i)} & =\frac{3}{8} \partial_{\rho} \ln \left(g_{Y Y}^{E(i)}\right) \partial_{z} \ln \left(g_{Y Y}^{E(i)}\right)
\end{aligned}
$$

Let us also note that, in general, the exchange of the two sigma models $M^{(1)} \longleftrightarrow$ $M^{(2)}$ leads to different EM solutions.

The presented proposition gives us a tool to generate new 5D EM solutions in a simple way from known solutions to the vacuum 5D Einstein equations. The technical difficulties are eventually concentrating in finding of $\Omega_{E}$ from (34) and $f_{E}$ from (39).

Through the use of the proposition we can generate the "5D EM images" of all known solutions of the vacuum 5D Einstein equations with the symmetries we consider here. It is not possible to present explicitly here the "EM images" of all known vacuum Einstein solutions. We shall demonstrate the application of the proposition on the case of rotating neutral black rings generating in this way a new rotating six parametric EM solution which includes he EM rotating dipole black ring solution as a particular case.

\section{Derivation of the rotating dipole black ring solution}

The rotating dipole black ring solutions in 5D Einstein-Maxwell-dilaton (EMd) gravity were given in 3] without any derivation. What is said in [3] is that these solutions can be obtained form generalized $C$-metric [31] by double Wick rotation and analytic continuation of parameters. As far as we are aware there is no explicit derivation of the dipole black ring solutions. Here we generate a new (six parametric) rotating EM solution and as a byproduct we give an explicit derivation of the EM rotating dipole black ring solution ${ }^{5}$.

The first step we should make in order to derive the EM dipole black ring solution is to chose two known solutions of the vacuum 5D Einstein equations and to present them in canonical coordinates. As should be expected, we take two copies of the neutral black ring solution with different parameters: the first solution is with parameters

\footnotetext{
${ }^{5}$ This solution can be obtained form the EMd dipole black ring solutions in the limit when the dilaton coupling parameter is zero.
} 
$\left\{\lambda_{1}, \nu_{1}, \mathcal{R}_{1}\right\}$ while the second is parameterized by $\left\{\lambda_{2}, \nu_{2}, \mathcal{R}_{2}\right\}$. It should be also noted that in the case under consideration the Killing vectors are denoted by

$$
K_{1}=\partial / \partial \psi, \quad K_{2}=\partial / \partial \phi .
$$

The neutral black ring solution has already been written in canonical coordinates in [32, that is why we present here the final formulas:

$$
\begin{aligned}
\left|g_{00}^{E(i)}\right|= & \frac{\left(1+\lambda_{i}\right)\left(1-\nu_{i}\right) R_{1}^{(i)}+\left(1-\lambda_{i}\right)\left(1+\nu_{i}\right) R_{2}^{(i)}-2\left(\lambda_{i}-\nu_{i}\right) R_{3}^{(i)}-\lambda_{i}\left(1-\nu_{i}^{2}\right) \mathcal{R}_{i}^{2}}{\left(1+\lambda_{i}\right)\left(1-\nu_{i}\right) R_{1}^{(i)}+\left(1-\lambda_{i}\right)\left(1+\nu_{i}\right) R_{2}^{(i)}-2\left(\lambda_{i}-\nu_{i}\right) R_{3}^{(i)}+\lambda_{i}\left(1-\nu_{i}^{2}\right) \mathcal{R}_{i}^{2}}, \\
g_{\Phi \Phi}^{E(i)}= & \frac{\left(R_{3}^{(i)}+z-\frac{1}{2} \mathcal{R}_{i}^{2}\right)\left(R_{2}^{(i)}-z+\frac{1}{2} \mathcal{R}_{i}^{2} \nu_{i}\right)}{R_{1}^{(i)}-z-\frac{1}{2} \mathcal{R}_{i}^{2} \nu_{i}} \\
= & \frac{\left(R_{1}^{(i)}+R_{2}^{(i)}+\nu_{i} \mathcal{R}_{i}^{2}\right)\left(R_{1}^{(i)}-R_{3}^{(i)}+\frac{1}{2}\left(1+\nu_{i}\right) \mathcal{R}_{i}^{2}\right)\left(R_{2}^{(i)}+R_{3}^{(i)}-\frac{1}{2}\left(1-\nu_{i}\right) \mathcal{R}_{i}^{2}\right)}{\mathcal{R}_{i}^{2}\left(\left(1-\nu_{i}\right) R_{1}^{(i)}-\left(1+\nu_{i}\right) R_{2}^{(i)}-2 \nu_{i} R_{3}^{(i)}\right)} \\
g_{\rho \rho}^{E(i)}= & {\left[\left(1+\lambda_{i}\right)\left(1-\nu_{i}\right) R_{1}^{(i)}+\left(1-\lambda_{i}\right)\left(1+\nu_{i}\right) R_{2}^{(i)}-2\left(\lambda_{i}-\nu_{i}\right) R_{3}^{(i)}+\lambda_{i}\left(1-\nu_{i}^{2}\right) \mathcal{R}_{i}^{2}\right] } \\
& \times \frac{\left(1-\nu_{i}\right) R_{1}^{(i)}+\left(1+\nu_{i}\right) R_{2}^{(i)}+2 \nu_{i} R_{3}^{(i)}}{8\left(1-\nu_{i}^{2}\right)^{2} R_{1}^{(i)} R_{2}^{(i)} R_{3}^{(i)}}, \\
\mathcal{A}_{E}^{(i)}= & \frac{-2 C\left(\nu_{i}, \lambda_{i}\right) \mathcal{R}_{i}\left(1-\nu_{i}\right)\left[R_{3}^{(i)}-R_{1}^{(i)}+\frac{1}{2} \mathcal{R}_{i}^{2}\left(1+\nu_{i}\right)\right]}{\left(1+\lambda_{i}\right)\left(1-\nu_{i}\right) R_{1}^{(i)}+\left(1-\lambda_{i}\right)\left(1+\nu_{i}\right) R_{2}^{(i)}-2\left(\lambda_{i}-\nu_{i}\right) R_{3}^{(i)}-\lambda_{i}\left(1-\nu_{i}^{2}\right) \mathcal{R}_{i}^{2}}
\end{aligned}
$$

where

$$
\begin{aligned}
R_{1}^{(i)} & =\sqrt{\rho^{2}+\left(z+\frac{\nu_{i}}{2} \mathcal{R}_{i}^{2}\right)^{2}}, \\
R_{2}^{(i)} & =\sqrt{\rho^{2}+\left(z-\frac{\nu_{i}}{2} \mathcal{R}_{i}^{2}\right)^{2}}, \\
R_{3}^{(i)} & =\sqrt{\rho^{2}+\left(z-\frac{1}{2} \mathcal{R}_{i}^{2}\right)^{2}}, \\
C\left(\nu_{i}, \lambda_{i}\right) & =\sqrt{\lambda_{i}\left(\lambda_{i}-\nu_{i}\right) \frac{1+\lambda_{i}}{1-\lambda_{i}}} .
\end{aligned}
$$

The next step is to find the functions $\Omega_{E}^{(i)}$ and $f_{E}^{(1)}$. After straightforward but tedious calculations we obtain

$$
\begin{aligned}
e^{\frac{8}{3} \Omega_{E}^{(i)}} & =\frac{\left[\left(1-\nu_{i}\right) R_{1}^{(i)}+\left(1+\nu_{i}\right) R_{2}^{(i)}+2 \nu_{i} R_{3}^{(i)}\right]^{2}}{8\left(1-\nu_{i}^{2}\right)^{2} R_{1}^{(i)} R_{2}^{(i)} R_{3}^{(i)}} g_{\Phi \Phi}^{E(i)}, \\
f_{E}^{(i)} & =\frac{\left(1-\nu_{i}\right) \mathcal{R}_{i} C\left(\nu_{i}, \lambda_{i}\right)\left[R_{1}^{(i)}-R_{3}^{(i)}+\frac{1}{2}\left(1+\nu_{i}\right) \mathcal{R}_{i}^{2}\right]}{\left(1+\lambda_{i}\right)\left(1-\nu_{i}\right) R_{1}^{(i)}+\left(1-\lambda_{i}\right)\left(1+\nu_{i}\right) R_{2}^{(i)}+2\left(\nu_{i}-\lambda_{i}\right) R_{3}^{(i)}+\lambda_{i}\left(1-\nu_{i}^{2}\right) \mathcal{R}_{i}^{2}} .
\end{aligned}
$$

Once having the functions $\Omega_{E}^{(i)}$ and $f_{E}^{(1)}$ in explicit form we can immediately apply the proposition and we obtain explicitly a new EM solution presented in the canonical 
coordinates. The found EM solution depends on six parameters $\left\{\lambda_{i}, \nu_{i}, \mathcal{R}_{i}, i=1,2\right\}$ and, obviously, the solution is very complicated. The detailed study of this new solution needs a separate investigation which we postpone for future publication. Here we will consider only the particular case when

$$
\nu_{1}=\nu_{2}=\nu, \quad \mathcal{R}_{1}=\mathcal{R}_{2}=\mathcal{R}
$$

In this case we also have

$$
\Omega_{E}^{(1)}=\Omega_{E}^{(2)}, \quad R_{a}^{(1)}=R_{a}^{(2)}, a=1,2,3
$$

which considerably simplifies the solution. Even in this particular case the solution looks complicated in the canonical coordinates. That is why it is more convenient to present the solution in coordinates where it takes simpler form. Such coordinates are the so-called $C$-metric coordinates given by

$$
\rho=\frac{\mathcal{R}^{2} \sqrt{-G(x) G(y)}}{(x-y)^{2}}, \quad z=\frac{1}{2} \frac{\mathcal{R}^{2}(1-x y)(2+\nu x+\nu y)}{(x-y)^{2}}
$$

where

$$
\begin{gathered}
G(x)=\left(1-x^{2}\right)(1+\nu x) \\
-1 \leq x \leq 1, \quad y \leq-1
\end{gathered}
$$

Performing this coordinate change we find

$$
\begin{aligned}
d s^{2}= & {\left[\frac{F_{\lambda_{1}}(y)}{F_{\lambda_{1}}(x)}\right]^{2} \frac{\mathcal{R}^{2} G(x)}{(x-y)^{2}} d \phi^{2}+\left[\frac{F_{\lambda_{1}}(x)}{F_{\lambda_{1}}(y)}\right]\left[-\frac{F_{\lambda_{2}}(y)}{F_{\lambda_{2}}(x)}\left(d t+C\left(\nu, \lambda_{2}\right) \mathcal{R} \frac{1+y}{F_{\lambda_{2}}(y)} d \psi\right)^{2}\right.} \\
& \left.-\frac{\mathcal{R}^{2} F_{\lambda_{2}}(x)}{(x-y)^{2}} \frac{G(y)}{F_{\lambda_{2}}(y)} d \psi^{2}+F_{\lambda_{1}}^{3}(y) \frac{\mathcal{R}^{2} F_{\lambda_{2}}(x)}{(x-y)^{2}}\left(\frac{d x^{2}}{G(x)}-\frac{d y^{2}}{G(y)}\right)\right] \\
A_{\phi}= & \pm \sqrt{3} C\left(\nu, \lambda_{1}\right) \mathcal{R} \frac{1+x}{F_{\lambda_{1}}(x)}+\text { const. }
\end{aligned}
$$

The metric can be rearranged into the form

$$
\begin{aligned}
d s^{2} & =-\frac{F_{\lambda_{2}}(y)}{F_{\lambda_{2}}(x)} \frac{F_{\lambda_{1}}(x)}{F_{\lambda_{1}}(y)}\left(d t+C\left(\nu, \lambda_{2}\right) \mathcal{R} \frac{1+y}{F_{\lambda_{2}}(y)} d \psi\right)^{2} \\
& +\left[F_{\lambda_{1}}(x) F_{\lambda_{1}}^{2}(y)\right] \frac{\mathcal{R}^{2} F_{\lambda_{2}}(x)}{(x-y)^{2}}\left[-\frac{G(x)}{F_{\lambda_{1}}^{3}(y) F_{\lambda_{2}}(y)} d \psi^{2}+\frac{d x^{2}}{G(x)}-\frac{d y^{2}}{G(y)}+\frac{G(x)}{F_{\lambda_{1}}^{3}(x) F_{\lambda_{2}}(x)} d \phi^{2}\right] .
\end{aligned}
$$

Finally, in order to exclude pathological behaviors of the metric we must consider only negative $\lambda_{1}$, i.e. 


$$
\lambda_{1}=-\mu, 0 \leq \mu<1 .
$$

and positive $\lambda_{2}$ and $\nu$ satisfying

$$
0<\nu \leq \lambda_{2}<1 .
$$

One can easily see that the generated 5D EM solution is just the EM rotating dipole black ring solution. Let us also recall[3] that in order to avoid the possible conical singularities at $x= \pm 1$ and $y=-1$ we must impose

$$
\begin{aligned}
& \Delta \phi=\Delta \psi=2 \pi \frac{(1+\mu)^{3 / 2} \sqrt{1-\lambda_{2}}}{1-\nu}, \\
& \frac{1-\lambda_{2}}{1+\lambda_{2}}\left(\frac{1+\mu}{1-\mu}\right)^{3}=\left(\frac{1-\nu}{1+\nu}\right)^{2} .
\end{aligned}
$$

\section{Conclusion}

In this paper we considered EM gravity in spacetimes admitting three commuting Killing vectors: one timelike and two spacelike one of them being hypersurface orthogonal. Assuming also a special ansatz for the electromagnetic field we have shown that the EM equations reduce to two $S L(2, R) / S O(2) \sigma$-models and a separated linear system of first order partial differential equations. This ensures the existence of Laxpair presentation, therefore the complete integrability of the considered sector of EM gravity. The Lax pair presentation also opens the way to apply the IST method and to generate multisoliton solutions.

Using the two $\sigma$-models structure of the reduced EM sector we gave an explicit construction for generating exact 5D EM solutions from known solutions of the 5D vacuum Einstein equations in the same symmetry sector. As an explicit example we constructed a six parameter rotating EM solution including as a particular case the rotating EM dipole black ring solution. In this way we gave, for the first time, an explicit derivation of the dipole black ring solution.

We shall conclude with some prospects for future work. Here we have shown that the "superposition" of two neutral black ring solutions with certain parameters yields the dipole EM black ring solution which schematically can be expressed as

$$
\{\text { neutral black ring }\}+\{\text { neutral black ring }\} \rightarrow\{E M \text { dipole black ring }\} .
$$

It would be interesting to find the EM solutions corresponding to the schemes

$$
\begin{aligned}
& \{\text { neutral black hole }\}+\{\text { neutral black hole }\} \rightarrow\{?\}, \\
& \{\text { neutral black hole }\}+\{\text { neutral black ring }\} \rightarrow\{?\}, \\
& \{\text { neutral black ring }\}+\{\text { neutral black hole }\} \rightarrow\{?\},
\end{aligned}
$$


as well as other solutions. Some solutions of vacuum 5D Einstein equations which could serve as seeds for new EM solutions are given in 32-36].

It would also be of interest to generalized this work for EM gravity in spacetimes with number of dimensions greater than five and in the presence of a dilaton field nonminimaly coupled to the electromagnetic field. Some results in these directions have already been found [37. They will be presented elsewhere.

\section{Acknowledgements}

I would like to thank I. Stefanov for reading the manuscript. This work was partially supported by the Bulgarian National Science Fund under Grant MUF04/05 (MU 408) and the Sofia University Research Fund.

\section{References}

[1] R. Emparan and H. Reall, Phys. Rev. D65, 084025 (2002).

[2] R. Emparan and H. Reall, Phys. Rev. Lett. 88, 101101 (2002).

[3] R. Emparan, JHEP 0403, 064 (2004).

[4] J. Kunz, F. Navarro-Lerida and A. Petersen, Phys. Lett. B614 , 104 (2005).

[5] J. Kunz and F. Navarro-Lerida, Phys. Rev. Lett. 96, 081101 (2006).

[6] R. Geroch, J. Math. Phys. 13, 394 (1972).

[7] W. Kinnersly, J. Math. Phys. 14, 651 (1973).

[8] W. Kinnersly, J. Math. Phys. 18, 1529 (1977).

[9] D. Maison, J. Math. Phys. 20, 871 (1979).

[10] I. Hauser and F. Ernst, Phys. Rev. D20, 362 (1979).

[11] I. Hauser and F. Ernst, Phys. Rev. D20, 1783 (1979).

[12] I. Hauser and F. Ernst, J. Math. Phys. 21, 1126 (1980).

[13] I. Hauser and F. Ernst, J. Math. Phys. 21, 1418 (1980).

[14] W. Kinnersly and D. Chitre, J. Math. Phys. 18, 1538 (1977).

[15] W. Kinnersly and D. Chitre, J. Math. Phys. 19, 1926 (1978).

[16] W. Kinnersly and D. Chitre, J. Math. Phys. 19, 1926 (1978).

[17] W. Kinnersly and D. Chitre, J. Math. Phys. 19, 2037 (1978). 
[18] D. Maison, Phys. Rev. Lett. 41, 521 (1978).

[19] B. Harrison, Phys. Rev. Lett. 41, 1197 (1978).

[20] G. Neugebauer, J. Phys. 12, L67 (1979).

[21] I. Bakas, Nucl. Phys. B428, 374 (1994).

[22] D. Gal'tsov, Phys. Rev. Lett. 74, 2863 (1995).

[23] A. Herrera-Aguilar and O. Kechkin, Int. J. Mod. Phys. A13, 393 (1998).

[24] A. Herrera-Aguilar and O. Kechkin, Mod. Phys. Lett. A13, 1979 (1998).

[25] A. Herrera-Aguilar and O. Kechkin, Phys. Rev. D59, 124006 (1999).

[26] M. Yurova, Phys. Rev. D64, 024022 (2001).

[27] D. Maison, Gen. Rel. Grav. 10, 717 (1979).

[28] A. Pomeransky, hep-th/0507250

[29] V. Belinski and V. Zakharov, Sov. Phys. JEPT 48, 985 (1978).

[30] V. Belinski and V. Zakharov, Sov. Phys. JEPT 50, 1 (1978).

[31] R. Emparan, Nucl. Phys. 490, 365 (1997).

[32] T. Harmark, Phys. Rev. D70, 124002 (2004).

[33] T. Mishima and H. Iguchi, hep-th/0504018.

[34] T. Koikawa, Prog. Theor. Phys. 114, 793 (2005).

[35] S. Tomizawa, Y. Morisawa and Y. Yasui, hep-th/0512252.

[36] T. Azuma and T. Koikawa, hep-th/0512350

[37] S. Yazadjiev, paper in preparation 